\title{
Análise da Gasometria e dos Níveis de Lactato Na Hipertensão Intra-abdominal Associada à Sepse Abdominal: Modelo Experimental em Ratos
}

\author{
Pedro Hurtado Rojas, Luana Alves Tannous, Luiz C Von Bahten, Felipe Castro Villegas, Juliano Gasparetto
}

\section{RESUMO}

Introdução: A hipertensão intra-abdominal e a sepse são condições clínicas de grande importância e alta prevalência em pacientes criticamente enfermos. O diagnóstico precoce permite um tratamento adequado e a melhora do prognóstico dos doentes.

Objetivo: O objetivo do estudo foi analisar os níveis de lactato e os componentes da gasometria em ratos submetidos à sepse e à hipertensão intra-abdominal.

Método: O experimento utilizou 40 ratos da linhagem Wistar divididos em 4 grupos: controle, grupo sepse, grupo hipertensão intra-abdominal e grupo sepse + hipertensão intra-abdominal. A sepse foi induzida pela ligadura e punção do ceco e a hipertensão intra-abdominal foi gerada pela injeção intraperitoneal de gelatina fluída modificada para manutenção de uma pressão de $15 \mathrm{~mm} \mathrm{Hg}$ durante o tempo de observação de 6 horas. Após esse período, uma dosagem de lactato e gasometria mista foram obtidas por punção intracardíaca e a eutanásia ocorreu por macrodose anestésica.

Resultados: Os resultados da comparação do grupo controle com o grupo hipertensão intra-abdominal e hipertensão intra-abdominal + sepse mostraram diferença significativa $(p<0,001)$ nos valores de $\mathrm{pH}$, excesso de base e bicarbonato, definindo acidose metabólica nos dois grupos do experimento. Não houve diferença dos níveis de lactato entre os grupos $(p>0,4)$. A comparação entre os parâmetros gasométricos do grupo controle e do grupo sepse não foi significativa para demonstrar acidose metabólica.

Conclusão: A gasometria é um marcador precoce e efetivo para hipoperfusão tecidual em ratos submetidos à hipertensão intra-abdominal.

Palavras-chave: Hipertensão intra-abdominal, Sepse, Gasometria, Lactato.

\section{ABSTRACT \\ Introduction: Intra-abdominal hypertension (IAH) and sepsis are great important clinical conditions and have a high prevalence in critically ill patients. The early diagnosis allows a proper treatment and improvement in patient's prognosis.}

Objective: This survey's purpose is to assay the lactate levels and analysis of blood gases components in rats that were subjected to sepsis and intra-abdominal hypertension.

Method: In the experiment, 40 Wistar rats were divided in 4 groups: control, sepsis group, intra-abdominal hypertension group and sepsis plus intra-abdominal hypertension group. Sepsis was induced by lashing and puncture of the cecum. The abdominal hypertension was caused by intraperitoneal injection of modified fluid gelatin to maintain a $15 \mathrm{~mm} \mathrm{Hg}$ pressure for
6 hours observation period. After this period, lactate dosage and mixed analysis of blood gases were obtained by cardiac puncture. The euthanasia occurred by anesthetic overdose.

Results: The comparison between the control group with the intra-abdominal hypertension and sepsis plus intra-abdominal hypertension groups demonstrated a significative difference $(p<0,001)$ of $\mathrm{pH}$ values, excess in base and bicarbonate levels, defining metabolic acidosis in both groups. The comparison between the analysis of blood gases parameters of control and sepsis groups was not significative $(p>0,4)$ to demonstrate metabolic acidosis.

Conclusion: Analysis of blood gases is an early and effective marker of tissue hypoperfusion in rats with intra-abdominal hypertension.

Keywords: Intra-abdominal hypertension, Sepsis, Analysis of blood gases, Lactate.

How to cite this article: Rojas $\mathrm{PH}$, Tannous LA, Von Bahten LC, Villegas FC, Gasparetto J. Análise da Gasometria e dos Níveis de Lactato Na Hipertensão Intra-abdominal Associada à Sepse Abdominal: Modelo Experimental em Ratos. Panam J Trauma Critical Care Emerg Surg 2013;2(1):49-51.

\section{Source of support: Nil}

Conflict of interest: None declared

\section{INTRODUÇÃO}

A HIA é definida como o aumento da pressão acima de $12 \mathrm{~mm} \mathrm{Hg}$ dentro da cavidade abdominal fechada. ${ }^{1,2}$ A SCA ocorre quando o aumento da pressão ameaça a viabilidade dos tecidos dentro do compartimento, levando à disfunção dos órgãos intra-abdominais e à repercussões devastadoras nos órgãos distantes. ${ }^{2,3}$

Reconhecer a HIA e SCA como condições ameaçadoras à vida é essencial para o tratamento. ${ }^{4}$ A detecção precoce e o tratamento apropriado diminuem significativamente a morbidade e mortalidade dos pacientes. ${ }^{1,3,5}$ A HIA é fator preditor independente de mortalidade nos pacientes críticos pelo seu importante papel na falência múltipla de órgãos. ${ }^{3}$

A HIA com repercussão clínica é observada em grande número e condições que são comuns nas unidades de terapia intensiva ${ }^{2,6,7}$ e seu diagnóstico e manejo têm ganho importância nesses centros. ${ }^{1}$ A sepse, sepse grave e choque séptico são situações de aumento da permeabilidade capilar manejadas com reposição volêmica vigorosa e são causas bastante prevalentes de HIA e SCA. ${ }^{2,8} \mathrm{~A}$ taxa de mortalidade

Paper presented at the Resident Competition, 25th Annual Congress of Panamerican Trauma Society, Medellin, Colombia, November 2012. 
da sepse grave e choque séptico são altos na maioria dos centros de terapia intensiva. ${ }^{9}$

Define-se como sepse grave a disfunção orgânica ou hipoperfusão tecidual decorrente de uma infecção, e, como choque séptico, a hipotensão associada à disfunção orgânica e hipoperfusão que não é revertida com reposição volêmica adequada. $^{9}$

A hipóxia tecidual ocorre quando a oferta de oxigênio não supre a demanda necessária, com consequente metabolismo anaeróbio e produção de lactato. A dosagem de lactato é um marcador diagnóstico, terapêutico e prognóstico da hipóxia tecidual global e choque circulatório. Elevações persistentes dos níveis de lactato estão associadas a elevada taxa de mortalidade. $^{10}$

A acidose metabólica é um fenômeno comum e clinicamente significativo em pacientes com sepse grave e choque séptico. A magnitude da acidose metabólica e da hipercloremia é maior entre os pacientes que não sobrevivem a uma infecção. Enquanto pacientes não sobreviventes mantêm a mesma magnitude da acidose metabólica e evoluem com queda progressiva do $\mathrm{pH}$ por retenção de dióxido de carbono, a melhora dos níveis de lactato e da acidose ao longo do internamento estão relacionados com a diminuição da mortalidade. ${ }^{11}$

Assim, um estudo experimental que correlaciona marcadores séricos de hipoperfusão como o lactato e alterações gasométricas, com duas situações clínicas tão graves e frequentes como a sepse e a HIA, pode ajudar a definir o comportamento fisiológico desses marcadores e estimular futuras pesquisas sobre esse assunto.

\section{OBJETIVO}

Analisar os níveis de lactato e os componentes da gasometria mista em modelos animais submetidos à sepse abdominal, hipertensão intra-abdominal e sepse associada à hipertensão intra-abdominal.

\section{MÉTODO}

O experimento utilizou 45 ratos da linhagem Wistar, 40 foram avaliados no presente estudo e os cinco demais foram utilizados para o ensaio piloto. $\mathrm{O}$ estudo piloto foi utilizado para ajustar a técnica operatória, testar as condições e doses corretas da anestesia e verificar o tempo correto de coleta e entrega dos exames laboratoriais.

Os 40 ratos do estudo foram divididos aleatoriamente em quatro grupos com 10 animais cada: $\mathrm{O}$ grupo $\mathrm{C}$ foi o grupo controle, que foi anestesiado e submetido à laparotomia exploradora, sem qualquer procedimento abdominal; grupo $\mathrm{S}$ foi o grupo da sepse abdominal submetido à laparotomia exploradora, ligadura do ceco e punção do ceco com agulha para indução de peritonite; o grupo HIA foi o grupo da hipertensão intra-abdominal, produzida pela injeção de gelatina fluída modificada por um cateter sobre agulha número dezoito gauge, puncionado na região do abdome superior do rato, conectado a um transdutor de pressão e fisiômetro para aferição da PIA, que foi mantida constante em $15 \mathrm{~mm} \mathrm{Hg}$; o grupo HIA+S foi o grupo sepse associado a HIA, com a realização de todos os procedimentos de forma conjunta submetido à laparotomia exploradora, ligadura do ceco e punção do ceco com agulha para indução de peritonite; o grupo HIA foi o grupo da hipertensão intra-abdominal, produzida pela injeção de gelatina fluída modificada por um cateter sobre agulha número dezoito gauge, puncionado na região do abdome superior do rato, conectado a um transdutor de pressão e fisiômetro para aferição da PIA, que foi mantida constante em $15 \mathrm{~mm} \mathrm{Hg}$; o grupo HIA+S foi o grupo sepse associado a HIA, com a realização de todos os procedimentos de forma conjunta.

Os animais foram mantidos anestesiados por seis horas, sem qualquer intervenção para manutenção da pressão arterial ou padrão ventilatório. Punção cardíaca foi realizada para a coleta de sangue e dosagem de lactato e gasometria. Após esse período, foi realizada a eutanásia com macrodose anestésica.

Em todos o os animais dos grupos HIA, para conter a expansibilidade excessiva da parede, o abdome do animal foi contido com fita adesiva do mesmo tamanho do perímetro abdominal prévio ao procedimento. Para colocação da fita, a circunferência abdominal foi medida e fita cortada com o dobro do tamanho dessa circunferência. A fita conteve toda a extensão do abdome, porém, sem englobar o tórax e atrapalhar a ventilação do animal.

Após a reunião de todos os dados testou-se a hipótese de que HIA e sepse levam ao aumento dos níveis de lactato e a alteração dos componentes da gasometria.

Os testes pareados realizados no mesmo animal (amostras dependentes) utilizaram o teste $t$ de Student.

\section{RESULTADOS}

Os resultados da comparação do grupo controle com o grupo hipertensão intra-abdominal e hipertensão intra-abdominal + sepse mostraram diferença significativa $(\mathrm{p}<0,001)$ nos valores de $\mathrm{pH}$, excesso de base e bicarbonato, definindo acidose metabólica nos dois grupos do experimento. Não houve diferença dos níveis de lactato entre os grupos $(p>0,4)$. A comparação entre os parâmetros gasométricos do grupo controle e do grupo sepse não foi significativa para demonstrar acidose metabólica (Tabla 1 to 3 ). 
Tabla 1: Média dos parâmetros em cada grupo do estudo

\begin{tabular}{lrrrr} 
& Grupo C & Grupo S & Grupo HIA & Grupo HIA+S \\
\hline Lactato $(\mu \mathrm{mol} / \mathrm{l})$ & 1,18 & 1,25 & 1,02 & 1,37 \\
$\mathrm{pH}$ & 7,27 & 7,25 & 6,98 & 7,03 \\
$\mathrm{HCO}_{3}(\mathrm{mEq} / \mathrm{l})$ & 25,4 & 32,3 & 14,9 & 17,2 \\
$\mathrm{BE}$ & $-2,38$ & $+2,58$ & $-17,32$ & $-14,39$ \\
$\mathrm{PCO}_{2}(\mathrm{~mm} \mathrm{Hg})$ & 57,0 & 75,4 & 63,8 & 66,0 \\
\hline
\end{tabular}

Tabla 2: Comparação entre o grupo $\mathrm{C}$ e o grupo HIA

\begin{tabular}{lrrc} 
& Grupo C & Grupo HIA & $p$ \\
Lactato $(\mu \mathrm{mol} / \mathrm{l})$ & 1,18 & 1,02 & 0,625 \\
$\mathrm{pH}$ & 7,27 & 6,98 & 0,000 \\
$\mathrm{HCO}_{3}(\mathrm{mEq} / \mathrm{l})$ & 25,4 & 14,9 & 0,000 \\
$\mathrm{BE}$ & $-2,38$ & $-17,32$ & 0,000 \\
$\mathrm{PCO}_{2}(\mathrm{~mm} \mathrm{Hg})$ & 57,0 & 63,8 & 0,378 \\
\hline
\end{tabular}

\begin{tabular}{lrrc}
\multicolumn{4}{c}{ Tabla 3: Comparação entre os grupos S e HIA } \\
& Grupo S & Grupo HIA & \multicolumn{1}{c}{$p$} \\
\hline Lactato $(\mu \mathrm{mol} / \mathrm{l})$ & 1,25 & 1,02 & 0,428 \\
$\mathrm{pH}$ & 7,25 & 6,98 & 0,000 \\
$\mathrm{HCO}_{3}(\mathrm{mEq} / \mathrm{l})$ & 32,3 & 14,9 & 0,000 \\
$\mathrm{BE}$ & $+2,58$ & $-17,32$ & 0,000 \\
$\mathrm{PCO}_{2}(\mathrm{~mm} \mathrm{Hg})$ & 75,4 & 63,8 & 0,152 \\
\hline
\end{tabular}

\section{CONCLUSÃO}

Após a análise dos dados, este estudo permite as seguintes conclusões:

- A análise da gasometria se mostrou um marcador precoce e efetivo de hipoperfusão tecidual em ratos submetidos à hipertensão intra-abdominal.

- Não houve aumento dos valores de lactato sérico nos animais testados para hipertensão intra-abdominal com níveis de $15 \mathrm{~mm} \mathrm{Hg}$.

\section{REFERENCIAS}

1. Malbrain ML, Chetham ML, Kirkpatrick A, Sugrue M, Parr M, De Waele J, et al. Results from the international conference of experts on intra-abdominal hypertension and abdominal compartment syndrome - I definitions. Intensive Care Med 2006;32(11):1722-32.

2. de Laet IE, Malbrain M. Current insights in intra-abdominal hypertension and abdominal compartment syndrome. Med Intensiva 2007;31(2):88-99.

3. Cheatham MI. Abdominal compartment syndrome: Pathophysiology and definitions. Scand J Trauma Ressusc Emerg Med 2009:17(1):10.

4. Eddy V, Nunn C, Morris JA. Abdominal compartment syndrome, the nashville experience. Surg Clin North Am 1997;77:801:12.
5. McNelis J, Marini CP, Simms HH. Abdominal compartment syndrome: Clinical manifestations and predictive factors. Curr Opin Crit Care 2003;9(2):133-36.

6. Hunter JD, Damani Z. Intra-abdominal hypertension and the abdominal compartment syndrome. Anaesthesia 2004;59: 899-907.

7. Morken J, West MA. Abdominal compartment syndrome in intensive care unit. Curr Op Crit Care 2001;7(4):268-74.

8. Wittmann DH, Iskander GA. The compartment syndrome of abdominal cavity: A state of art review. J Intensive Care Med 2000;15:201-20.

9. Dellinger RP, Carlet JM, Masur H, Gerlach H, Calandra T, Cohen $\mathrm{J}$, et al. Surviving Sepsis Campaign guidelines for management of severe sepsis and septic shock. Crit Care Med 2004;32(3): 858-73.

10. Casey LC, Balk RA, Bone RC. Plasma cytokine and endotoxin levels correlate with survival in patients with the sepsis syndrome. Ann Intern Med 1993;119(8):771-78.

11. Noritomi DT. Caracterização físico-química da acidose metabólica em pacientes com sepse grave e choque séptico [tese de doutorado]. São Paulo: Faculdade de Medicina da Universidade de São Paulo 2009.

\section{ACERCA DE LOS AUTORES}

\section{Pedro Hurtado Rojas (Autor Para Correspondencia)}

Pontifícia Universdade Católica Do Paraná, Centro De Ciências Biológicas E Da Saúde, Programa De Pós-Graduação Em Cirurgia Curitiba, e-mail: pedro2303@hotmail.es

\section{Luana Alves Tannous}

Pontifícia Universdade Católica Do Paraná, Centro De Ciências Biológicas E Da Saúde, Programa De Pós-Graduação Em Cirurgia Curitiba

\section{Luiz C Von Bahten}

Pontifícia Universdade Católica Do Paraná, Centro De Ciências Biológicas E Da Saúde, Programa De Pós-Graduação Em Cirurgia Curitiba

\section{Felipe Castro Villegas}

Pontifícia Universdade Católica Do Paraná, Centro De Ciências Biológicas E Da Saúde, Programa De Pós-Graduação Em Cirurgia Curitiba

\section{Juliano Gasparetto}

Pontifícia Universdade Católica Do Paraná, Centro De Ciências Biológicas E Da Saúde, Programa De Pós-Graduação Em Cirurgia Curitiba 